\title{
Chronic Mucocutaneous Candidiasis in Autoimmune Polyendocrine Syndrome Type 1
}

\begin{abstract}
Linda Humbert ${ }^{1}$, Marjorie Cornu ${ }^{2,3}$, Emmanuelle Proust-Lemoine ${ }^{4}$, Jagadeesh Bayry ${ }^{5}$, Jean-Louis Wemeau ${ }^{1}$, Marie-Christine Vantyghem ${ }^{1,6,7}$ and Boualem Sendid ${ }^{2,3 *}$

${ }^{1}$ Department of Endocrinology and Metabolism, CHU Lille, Lille, France, ${ }^{2}$ Department Parasitology-Mycology, CHU, Lille, France, ${ }^{3}$ Inserm, U995-LIRIC, Fungal Associated Invasive \& Inflammatory Diseases, Lille, France, ${ }^{4}$ Department of Endocrinology, Polyclinique Aguilera, Biarritz, France, ${ }^{5}$ Inserm, Center de Recherche des Cordeliers, Sorbonne Université, Université Paris Descartes, Sorbonne Paris Cité, Paris, France, ${ }^{6}$ UMR 1190, Translational Research in Diabetes Inserm, Lille, France, ${ }^{7}$ European Genomic Institute for Diabetes, Univ Lille, Lille, France
\end{abstract}

Autoimmune polyendocrinopathy candidiasis ectodermal dystrophy (APECED) is an autosomal recessive disease caused by mutations in the autoimmune regulator (AIRE) gene, characterized by the clinical triad of chronic mucocutaneous candidiasis (CMC), hypoparathyroidism, and adrenal insufficiency. CMC can be complicated by systemic candidiasis or oral squamous cell carcinoma (SCC), and may lead to death. The role of chronic Candida infection in the etiopathogenesis of oral SCC is unclear. Long-term use of fluconazole has led to the emergence of Candida albicans strains with decreased susceptibility to azoles. CMC is associated with an impaired Th17 cell response; however, it remains unclear whether decreased serum IL-17 and IL-22 levels are related to a defect in cytokine production or to neutralizing autoantibodies resulting from mutations in the AIRE gene.

Keywords: autoimmune polyendocrinopathy candidiasis ectodermal dystrophy (APECED), chronic mucocutaneous candidiasis (CMC), autoimmune regulator (AIRE) gene, IL-17, IL-22

\section{INTRODUCTION}

Autoimmune polyendocrine syndrome type 1 (APS-1), also known as autoimmune polyendocrinopathy candidiasis ectodermal dystrophy (APECED) syndrome, is characterized by the clinical triad of chronic mucocutaneous candidiasis (CMC), hypoparathyroidism, and adrenal insufficiency. This syndrome was formerly known as Whitaker syndrome (1). Accurate diagnosis of this syndrome requires the presence of at least two of these three major components, or only one if a sibling has already been diagnosed with the disease (2). Other autoimmune disorders have also been described, such as hypergonadotrophic hypogonadism, thyroid disease, type 1 diabetes, coeliac disease, liver disease, alopecia, vitiligo, chronic atrophic gastritis, and hypophysitis. These autoimmune disorders are associated with ectodermal dystrophy, asplenia, and the presence of several autoantibodies, even in the absence of corresponding organ dysfunction (3).

APS-1 is a monogenic, autosomal, recessive disease caused by a mutation in the autoimmune regulator (AIRE) gene on chromosome 21 (gene map locus 21q22.3) (4). The AIRE gene is composed of 14 exons and codes for a 545 amino acid protein $(5,6)$. The AIRE gene is mainly expressed in thymic medullary epithelial cells, which play an important role in the presentation of self-antigens $(7,8)$, but is also expressed at low levels in the spleen, lymph nodes, pancreas, adrenal cortex, and peripheral blood mononuclear cells. The AIRE gene codes for a nuclear 
transcriptional regulator protein involved in the ectopic expression of self-antigens in the thymus, leading to the removal of self-reactive thymocytes and generation of peripheral tolerance. The role of peripheral AIRE expression, which has been confirmed by mRNA analysis, remains unclear. To date, more than 100 different mutations in this gene, both homogeneous and heterogeneous, have been reported worldwide (9-12).

APECED is a rare syndrome, which has been reported worldwide, but is more prevalent in some historically-isolated homogeneous populations in Finland $(1 / 25000)(4,13)$, Sardinia $(1 / 14500)(14)$, and Iranian Jews (1/9000) (15). APECED is also seen at a lower incidence in Norway, Sweden, Slovenia, Great Britain, Italy, Ireland, and North America (16-21).

Patients with APS-1 suffer from CMC without displaying susceptibility to any other pathogen. CMC is associated with the Finnish mutation c.769C $>$ T (p.Arg257stop) (22). CMC usually affects the oral mucosa, but the nails and skin may also be involved. Esophageal candidiasis results in pain and dysphagia. CMC can be complicated by systemic candidiasis or oral squamous cell carcinomas (SCCs), and may lead to death $(23,24)$.

\section{CHRONIC MUCOCUTANEOUS CANDIDIASIS}

CMC is characterized by recurrent or persistent symptomatic mucocutaneous infections caused by Candida species, predominantly Candida albicans, affecting the nails, skin, oral cavity, and genital mucosa. The diagnosis of CMC is based on clinical symptoms, associated in most cases with the isolation of Candida from body sites (25). The first case of syndromic CMC was described by Thorp and Handley (26).

C. albicans is a ubiquitous, diploid, dimorphic yeast that resides as a commensal organism on the mucosae and in the gastrointestinal tract of healthy individuals. Mucosal candidiasis results from a change in mucosal homeostasis leading to disequilibrium between the yeast and its host. Opportunistic mucosal infection, deep organ, or systemic infection in immunocompromised patients usually arise from Candida colonizing the digestive tract (27). Systemic candidiasis can be diagnosed using a number of non-culture based assays (28) but no biological markers are currently available for the diagnosis of "culture-negative" CMC.

Most cases of CMC are sporadic and are secondary to other medical conditions such as HIV infection with T-cell deficiency, diabetes, immunosuppressive therapies like anticytokine blockers, antibiotic or steroid therapy $(25,29,30)$. CMC is also more rarely favored by genetic disorders (i.e., familial $\mathrm{CMC}$ ) that can be inherited. These cases of primary CMC are due mainly to innate immunodeficiency disorders. They have been reviewed by Puel et al. (31) and were classified as primary immunodeficiency disease by the International Union of Immunological Societies Expert Committee for Primary Immunodeficiency in 2015 (32).
Three types of immnuodeficiency can be distinguished depending on the genetic abnormalities associated with CMC:

(i) Severe underlying immunodeficiency (ID), including severe combined immunodeficiency (SCID), or CD25 deficiency. SCID is a body of diseases characterized by the inability to produce T-cells leading to Th17 cell deficiency $(32,33)$. Individuals with SCID are susceptible to a whole range of infections caused by bacteria and viruses. Subjects with autosomal recessive CD25 deficiency have a decrease in T-cell and Th17 cell numbers, and a consequent high incidence of viral and bacterial infections $(31,33)$.

(ii) HIES, CARD9, IL12Rb1 deficiency, GOF-STAT1, and APECED/APS1 are syndromes where CMC has additional specific clinical features. Autosomal dominant hyper $\operatorname{IgE}$ syndrome (AD-HIES) is characterized by impaired production of Th17 cells and Th17-derived cytokines caused by an autosomal dominant mutation of $\operatorname{STAT3}(31,34)$. ADHIES is associated with several infectious diseases, including staphylococcal skin abscesses, bacterial pneumonia, and CMC. Recent literature has also described an autosomal recessive hyper-IgE syndrome (AR-HIES), caused by a deficiency in DOCK8, resulting in a variety of symptoms and diseases including atopy, autoimmunity risk, malignancies, recurrent viral/bacterial infections, and CMC. Patients who are deficient in DOCK8 have decreased Th17 cells (35-38). Low concentrations of critical candidacidal peptides, including histatins and $\beta$-defensin 2 (BD2), which are activated by IL17 , are found in the saliva of individuals with HIES. Saliva from healthy individuals usually contains high concentrations of $\beta$-defensins and histatins, which have direct candidacidal activity. IL-17A, but not IL-22, acts directly on human salivary glands, which might explain why saliva from HIES patients is deficient in histatins and the increased susceptibility of these individuals to CMC. Antimicrobial peptides are secreted by skin epithelial cells only when they are stimulated with Th17 cytokines and classical proinflammatory cytokines (e.g., TNF$\alpha$, IL-1 $\beta$, IFN- $\gamma$ ) (34). Autosomal recessive tyrosine kinase deficiency is another type of combined immunodeficiency that mimics the symptoms of AR-HIES (31-33).

CMC and invasive Candida infection have also been associated with a deficiency in autosomal recessive caspase recruitment domain-containing protein 9 (CARD9) (39-41). CARD9 is an intracellular adaptor involved in Dectin 1 and Dectin 2 signaling, the main pathogen recognition receptors for $C$. albicans glycans. The number of circulating IL-17-producing cells and IL-17 secretion have been reported to be decreased in CARD9-deficient patients $(40,42,43)$. However, these findings remain open to debate (33). With regard to Candida infections, mutations in Dectin- 1 and Dectin- 2 have also been studied in murine models. In humans, a mutation in the early-stop codon for Dectin-1 (Y238X) has been reported in a family with recurrent vulvovaginal candidiasis. Experiments in vitro demonstrated that monocytes and neutrophils from homozygous patients lacking Dectin-1 expression are defective in cytokine production, including IL-17, when 
stimulated with C. albicans. However, phagocytosis and yeast cell killing remained normal $(44,45)$. Another report demonstrated that patients receiving hematopoietic stem cell transplants who were heterozygous for Y238X had an increased incidence of gastrointestinal Candida colonization (46). Although Dectin-2-deficient mice had higher mortality and a higher kidney fungal burden after infection with $C$. albicans (47), the impact of Dectin-2 mutations on the human host response to $C$. albicans infection remains unclear.

Other studies have demonstrated that patients with IL-12 or IL-23 signaling defects have an increased risk of developing CMC (31, 33, 48). Patients with CMC, autoimmune manifestations, other mild bacterial or viral infections, intracranial aneurysms, or SCC also had heterozygous missense gain-of-function (GOF) mutations of STAT1. The development of IL-17-producing T-cells is impaired in these patients, as a result of hyperactivity of STAT1 that inhibits STAT3 signaling. This phenomenon results in increased STAT1-dependent cellular responses that repress IL-17producing T-cell responses, such as IFN- $\gamma$, and/or enhanced IL-6, IL-21, and IL-23 STAT1 responses, which normally activate STAT3 and induce IL-17 T-cell production (31).

(iii) Isolated CMC has also been described in subjects with IL17RA, IL-17RC, ACT1, IL-17F, and ROR $\gamma$ t deficiency, where $\mathrm{CMC}$ is the only presenting feature of the disease. Some families have also been identified with autosomal-dominant mutations in the gene coding for IL-17F, or autosomalrecessive mutations in the gene coding for IL-17 receptor $\mathrm{A}$ or IL-17 receptor C as risk factors for CMC (31-33).

These familial cases of CMC demonstrate that IL-17 plays a pivotal role in human epithelial immunity to C. albicans. Another piece of evidence supporting the central role of Th17 cytokines in CMC and mucosal immunity to yeasts is the observation of cases of CMC in the Phase 2 trial of secukinumab, a human anti-IL17 receptor antibody for the treatment of Crohn's disease $(49,50)$. This review aims to focus on the characteristics of familial CMC associated with APECED syndrome.

\section{CHRONIC MUCOCUTANEOUS CANDIDIASIS AND APECED}

\section{Clinical Description}

CMC is the most common infection occurring in APECED patients $(77-100 \%)(19,21,23,51-53)$, except in Iranian Jews (17\%) (15). CMC is also the most common first clinical manifestation of APECED syndrome (40-93\%) (20, 23, 52, $54,55)$. Median age at diagnosis is usually $<5$-years-old (1.06.5 years $)(20,21,23,54,55)$. According to the Finnish series, one-sixth of patients had developed CMC by 1.0 year, half by 5 years, $70 \%$ by 10 years, $94 \%$ by 20 years, and $97 \%$ by 30 years of age (56).

The clinical course of CMC varies from periodic to chronic, and its severity varies between individuals. The oral cavity was involved in $100 \%$ of patients in the Finnish cohort (23). In the Norwegian cohort, $40 \%$ of patients had angular cheilitis (53).
In the mild oral form, CMC causes ulceration, redness, and soreness of the corners of the mouth. In more severe cases, the entire mouth is involved making it impossible to consume acidic or spicy foods. In the hyperplastic form, the tongue and buccal mucous membranes are covered by white or gray plaques and hyperkeratosis. In the atrophic form, the mucosa is erythematous and may be speckled with areas of leukoplakic or nodules (56). Candida onychomycosis is often associated with mucosal Candida lesions in childhood and is very difficult to eliminate (56). CMC affected the nails in $72 \%$ of patients in the Irish cohort of Collins et al. (52), and less frequently the skin (10$17 \%)(21,23)$. Esophageal CMC occurred in 5-22\% of patients in a European series $(23,52,53)$, and in $51 \%$ of patients in a recent American study (21). Esophageal candidiasis often occurs without the typical form or symptoms of oral candidiasis, and can be complicated by substernal pain, dysphagia, and stenosis. Esophageal stenosis requires endoscopic dilation (23, 52-54). In the digestive tract, CMC can cause abdominal pain, flatulence, and diarrhea, which may be severe. Symptomatic intestinal candidiasis may also be present in the absence of oral disease (56).

In some patients, CMC may also be complicated by systemic candidiasis, although evidence is lacking that dissemination occurs from the oral cavity. Systemic candidiasis is very rare, even in APECED patients, and is frequently associated with immunosuppressive therapy. In an Italian cohort of 41 patients, one patient died from systemic candidiasis after the onset of immunosuppressive treatment (54), and in a French cohort, one patient died from systemic candidiasis after the onset of immunosuppressive treatment for large granular lymphocytic leukemia (57). In a Finnish cohort, one patient developed an abscess on the pericardium and small intestine (23). One isolated case of chronic Candida otitis has been described (53).

\section{Genotype-Phenotype Correlation}

The prevalence of CMC is reportedly higher in patients with the major Finnish AIRE mutation R257X than with other mutations (22). The prevalence is $<20 \%$ in Iranian Jews affected by the Y85C mutation (15). Kisand et al. studied 160 APECED patients with the most severe mutations, R257X (Finnish) and R139X (Sardinian) and the Norwegian mutation, 967-979 13 In contrast to the study of Puel et al. (58), CMC was less prevalent in patients with the homozygous mutation 967-979 $\Delta 13$ than with the other two mutations (59).

\section{Squamous Cell Carcinoma and CMC}

CMC has been reported to be involved in carcinogenesis as cancer often develops at the site of fungal lesions. Several cases of oral carcinoma have been described in association with CMC of the oral cavity and esophagus, suggesting that oral candidiasis may be carcinogenic. The most common morphological entity of these cancers is $\operatorname{SCC}(29,60,61)$. In patients with chronic Candida infection, oral CMC is often associated with esophageal cancer (62), and good clinical practice should include regular monitoring, every 2-3 years, by endoscopy. CMC is induced by immunosuppressive therapy rather than SCC itself.

The first report of oral SCC associated with APECED syndrome was published in 1975 in a patient who died of 
metastatic disease at 27-years of age (63). A case report published in 2010 recorded the presence of three separate oral SCCs in a 40-year-old woman with APECED (61). In the Finnish case series, 6/91 APECED patients $>25$-years of age $(10.5 \%)$ developed SCC, four died from the disease, and two developed oral colonization with Candida with decreased susceptibility to azole antifungals. One patient developed SCC without symptoms of oral candidiasis $(23,60)$. In the Norwegian cohort, 3/52 patients (6\%) developed SCC at an early age (53). SCC was not reported in a recent American case series (21).

In the general population, mean age at diagnosis for oral and esophageal SCC is $>62$-years, and the disease is uncommon in young adults (64). The patients diagnosed with oral SCC in the Finnish cohort were between 29- and 44-years of age (23). The high rate of oral SCC in young patients with APECED demonstrates the possible carcinogenic potential of $C$. albicans when associated with the specific immunodeficiencies characteristic of this syndrome. Therefore, appropriate screening and adequate management of the infection and areas of oral dysplasia is necessary to reduce the risk of malignancy. Each erosive, ulcerated lesion should be biopsied, and each dysplastic lesion should be treated surgically (65).

In the general population, there are many risk factors for oral and esophageal SCC. Extrinsic factors include alcohol, tobacco, betel quid, immunosuppression, radiation, oncogenic viruses (human papilloma virus), and Candida infection, while intrinsic factors include immune defects, iron or vitamin A deficiency, malnutrition, and defects in tumor suppressor genes $(64,66,67)$. Of the six APECED patients who developed SCC in the Finnish cohort, four were smokers and one had received immunosuppressive therapy (23). Therefore, extrinsic factors for SCC should be controlled in APECED patients as well as in CMC.

The role of chronic Candida infection in the etiopathogenesis of oral SCC is unclear. Possible mechanisms by which oral Candida infection might contribute to cancer development include: (i) metabolism of procarcinogens (such as the conversion of ethanol to acetaldehyde by Candida); (ii) production of carcinogens (such as the production of nitrosamine by Candida species); and (iii) induction of chronic inflammation, with the production of cytokines that enhance cell proliferation and inhibit apoptosis $(24,64,65,68,69)$.

\section{C. albicans and Decreased Susceptibility to Antifungal Treatment}

Oropharyngeal candidiasis (mainly C. albicans and C. glabrata) is the most common fungal infection in patients with human immunodeficiency virus (HIV), and long-term use of azoles in this population has been reported to cause loss of susceptibility of C. albicans to fluconazole $(70,71)$. Candidiasis is usually caused by the yeast $C$. albicans in APECED patients, unlike in HIV patients $(23,52,72-75)$. In a group of Finnish patients with APECED, non-C. albicans spp. were reported in only 7/56 patients (12.5\%) (72). Because of the high prevalence of CMC in APECED patients and the risk of SCC, lifelong management of candidiasis with antifungal treatment is necessary (76). Topical intermittent treatment is more frequently prescribed than systemic antifungals, which are restricted to periods of severe symptoms and systemic candidiasis. Unlike in HIV patients, APECED patients with CMC treated with fluconazole have a high risk of becoming colonized with C. albicans with decreased susceptibility to fluconazole (76). Emergence of resistance does not appear to be a problem during short-term use, as shown in 43 isolates of $C$. albicans from 23 Finnish APECED patients.

Resistance seems to be correlated to the number of years of antifungal drug use, and is mainly due to the use of triazoles. Rautemaa et al. identified C. albicans isolates with decreased susceptibility to fluconazole in 11/56 (20\%) Finnish patients (72). In the Irish cohort study of 16 patients with APECED, McManus described 11/16 (69\%) patients with clinical signs of oral Candida infection and oral Candida isolates were recovered from 12/16 (75\%) patients. Surprisingly, clinical signs suggestive of candidiasis did not always correlate with microbiological evidence of infection, and yeasts were isolated from only $32 \%$ of patients. The susceptibility of sequentially recovered isolates to fluconazole and itraconazole was examined; 11/16 (69\%) patients had received antifungal treatment with fluconazole or itraconazole. Four of these 11 patients (36\%) had isolates that were resistant or had reduced susceptibility to azoles (74).

Multiple mechanisms could lead to azole resistance in $C$. albicans. Some genes of C. albicans involved in ergosterol synthesis can be upregulated and in this way confer resistance to azoles, in particular the ERG11 gene and CDR1, CDR2, and MDR1 encoded efflux pumps. In the study of McManus, acquisition of such mutations was revealed in C. albicans strains isolated during the longitudinal follow-up of patients. C. albicans strains recovered sequentially from nine Finnish patients were studied. The major molecular mechanisms leading to azole resistance were GOF mutations in TAC1, contributing to overexpression of CDR1 and CDR2, point mutations in ERG11, and six new TAC1 mutations were detected (77). To avoid the emergence of azole resistance, McManus et al. proposed only prescribing azole therapy in patients with mycologicallydocumented Candida infection.

\section{How Should CMC Be Managed in APECED Patients?}

Husebye et al. suggested that oral infection should be strictly controlled to prevent the development of cancer. These authors highlighted the importance of having good oral hygiene with abstention from smoking and excessive alcohol consumption, and to avoid eating acidic or spicy food, and toothpastes containing powerful whitening agents. Meticulous oral hygiene is recommended in CMC patients, using both toothpaste and chlorhexidine solution, at bedtime and long-term, with the continual use of two topical polyene drugs. In line with this recommendation, patients should hold $1-2 \mathrm{~mL}$ of nystatin suspension in their mouth for several minutes and then allow an amphotericin B lozenge to dissolve without chewing. Both drugs should be spread to every part of the mouth with the tongue and finally swallowed. This regimen should be continued for 4-6 weeks or for at least 1 week after the resolution of symptoms. The use of these two polyene antifungal drugs is important in clinical 
practice and both are well-tolerated due to a lack of absorption from the gut and a low rate of yeast resistance to these drugs.

Azole agents should be restricted to 2-3 courses per year to avoid decreased susceptibility. This treatment can be followed by prophylactic treatment consisting of 1 week of a polyene antifungal every 3 weeks and 1 week of chlorhexidine mouth rinse twice a day, if CMC becomes recurrent. Prophylactic treatment should be administered more frequently if symptoms persist, and even become daily treatment. In the case of failure of antifungal therapy, it is necessary to alert microbiologists to the possibility of a mixed infection and to use chromogenic media to detect mixed species, which would not be picked up with classical Sabouraud glucose agar. It is also recommended that all species isolated undergo antifungal susceptibility testing so that treatment can be adapted accordingly. Angular cheilitis should be treated by application of natamycin, amorolphine hydrochloride cream, or chlorhexidine gel several times a day, continuing for 4-5 days after the corners of the mouth have healed. Miconazole gel can also be used, while bearing in mind the risk of selection of azole-resistant strains. In order to avoid CMC relapses, antifungal treatment should be prescribed simultaneously for oral candidiasis and angular cheilitis, and biopsy of any lesion should be considered if mucositis with ulceration fails to respond to treatment within 2 weeks. Candida esophagitis and digestive $\mathrm{CMC}$ with diarrhea should also be treated with the same drug regimen for 1 or 2 weeks. If esophagitis persists, esophagoscopy and mycological sampling should be performed. The use of highdose fluconazole (200-300 mg once a day for 1 week) must be restricted to severe cases and failure of topical therapy. Vaginal Candida infection should be treated with a short course of vaginal fluconazole, while fingernail candidiasis is very hard to eliminate and systemic medication is often necessary for 6 weeks. In general terms, prolonged intravenous (IV) antifungal therapy may be required and administered after obtaining expert medical advice $(56,75)$.

Adjunct immunotherapy with normal pooled immunoglobulin or IV immunoglobulin $G$ (IVIG) in combination with antifungal agents is another treatment option. IVIG is a pooled IgG therapeutic preparation obtained from the plasma of several thousand healthy donors. In addition to its safety profile, IVIG could benefit CMC-associated APECED as a result of multiple mechanisms, including a reduction of inflammation by targeting various arms of the immune system, inhibition of autoantibody production by B-cells, and B-cell anergy (78). Further, antibodies in IVIG to fungal antigens may also help in pathogen neutralization $(79,80)$. As high-dose IVIG $(1-2 \mathrm{~g} / \mathrm{kg})$ is known to inhibit Th17 responses in vitro $(81,82)$ and in vivo, including experimental allergic bronchopulmonary aspergillosis $(78,83)$, studies are required regarding appropriate dose of IVIG for CMC-associated APECED.

\section{Pathophysiology of CMC Associated With APECED}

The most prevalent autoantibodies detected in APECED patients are those neutralizing cytokines, especially type I IFNs and

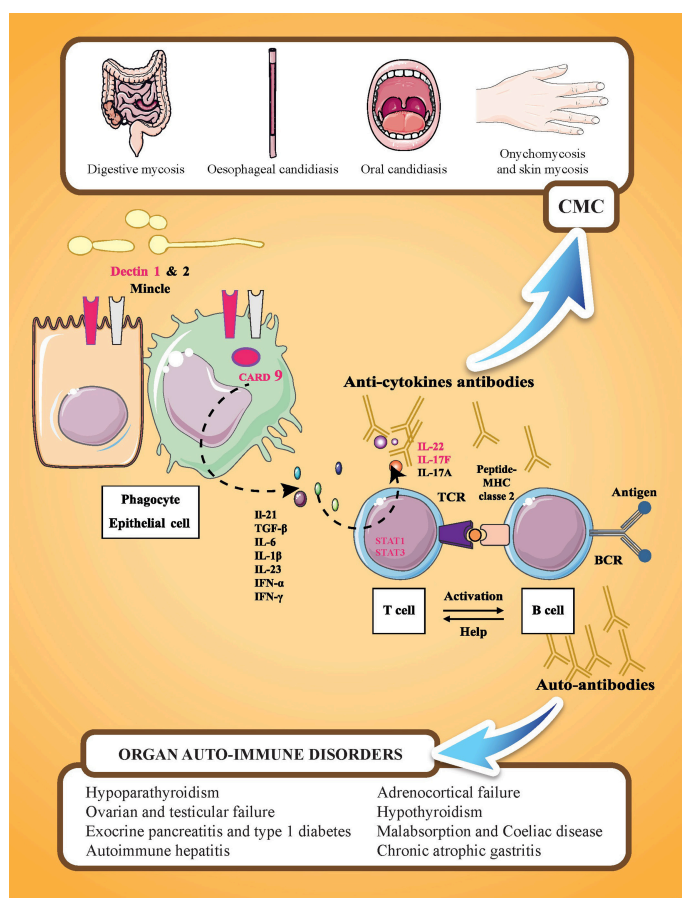

FIGURE 1 | Familial CMC and APECED syndrome. APECED syndrome is characterized by the association of endocrine autoimmune disorders (such as hypoparathyroidism, hypothyroidism, adrenocortical insufficiency, and gonada failure), non-endocrine autoimmune disorders (such as autoimmune hepatitis, celiac disease, and chronic atrophic gastritis), and chronic mucocutaneous candidiasis $(\mathrm{CMC})$. These manifestations are related to the presence of tissue specific antibodies and cytokine antibodies. IL-17 mediated immunity is represented by cooperation between cells recognizing $C$. albicans (phagocytes and epithelial cells) and IL-17 cytokine-producing cells (T-cells). On C. albicans recognition by PRRs (pathogen recognition receptors; including Dectin-1, Dectin-2, or Mincle), the adaptor molecule CARD9 mediates the induction of pro-inflammatory cytokines, such as IL $1 \beta, I L-6$, and IL-23. On binding to their receptors expressed on T-lymphocytes, pro-inflammatory cytokines, such as IL-6 or IL-23, activate T-lymphocytes via the transcription factor STAT3 resulting in their differentiation into IL-17-producing T-cells. Genes in which mutations are associated with $\mathrm{CMC}$ are indicated in pink: dectin 1, CARD 9, STAT 1, STAT3, IL22, and IL17F. IL-17RA and IL-12RB1 are not represented. $Y$ designates cytokine-neutralizing autoantibodies that develop in AIRE-deficient (APECED and rare thymoma cases) patients.

TH17-related cytokines, with a prevalence of $100 \%$ for IFN$\omega(84,85)$ and $>90 \%$ for IL-22. IFN- $\omega$ autoantibodies are also found in thymomas (86), with a high rate of specificity and sensitivity. These autoantibodies are highly disease specific. Antibodies neutralizing IFN- $\omega$ are not correlated with CMC, and high titers of anti-IFN- $\omega$ antibodies are found in patients without CMC. Antibody titers in patients with APECED are almost always higher at diagnosis and persist for decades, representing a reliable biomarker for APECED syndrome $(84,85)$. Neutralizing autoantibodies against IL-17A, IL-17F, and IL-22 are also present at diagnosis. Autoantibodies against IL-22 and IL-17F seem to be more prevalent in APECED patients with CMC than in those without CMC, suggesting that type Th17 cytokines are central in human epithelial immunity against Candida infection (Figure 1). In contrast to the study of Kisand et al. (59) who reported 
that autoantibodies neutralizing IL-22 and IL-17F (but not those against IL-17A) were correlated with CMC in a study of 162 APECED patients, a study by Sarkadi et al. (87) reported high levels of autoantibodies against IL-17A in APECED patients with severe CMC. However, recent experimental data have shown that autoantibodies against IL-17A that develop in older AIREdeficient mice do not confer susceptibility to oropharyngeal candidiasis, while monoclonal antibodies that cross-react with murine IL-22 derived from patients increase the mucosal fungal burden (88).

Occasional or weakly binding autoantibodies against IL6, IL-9, IL-12, IL-21, IL-23, IL-26, IL-29, and RANTES have been reported in APECED patients in addition to autoantibodies against Th17 cytokines; however, their role in the development of CMC has not been demonstrated clearly (59).

The production of type I IFNs by dendritic cells is not impaired in APECED patients (89). In vitro, IL-22 and IL$17 \mathrm{~F}$ production by peripheral blood mononuclear cells of APECED patients, stimulated by Candida antigens or polyclonal stimuli, was decreased in patients with $\operatorname{CMC}(59,90,91)$. The production of IL-17A was not impaired and even appeared to be increased $(90,91)$. The pathogenesis of CMC is believed to be associated with impaired Th17 cell responses, similar to several other primary immunodeficiencies associated with CMC. Th17 cytokines (IL-17A, IL-17F, and IL-22) influence epithelial cells by inducing the production of chemokines and antimicrobial peptides that exert direct antifungal activity. Additionally, IL-22

\section{REFERENCES}

1. Whitaker J, Landing BH, Esselborn VM, Williams RR. The syndrome of familial juvenile hypoadrenocorticism, hypoparathyroidism and superficial moniliasis. J Clin Endocrinol Metab. (1956) 16:1374-87.

2. Neufeld M, Maclaren N, Blizzard R. Autoimmune polyglandular syndromes. Pediatr Ann. (1980) 9:154-62.

3. Proust-Lemoine E, Saugier-Veber P, Wémeau J-L. Polyglandular autoimmune syndrome type I. Presse Méd. (2012) 41:e651-62. doi: 10.1016/j.lpm.2012.10.005

4. Aaltonen J, Björses P, Sandkuijl L, Perheentupa J, Peltonen L. An autosomal locus causing autoimmune disease: autoimmune polyglandular disease type I assigned to chromosome 21. Nat Genet. (1994) 8:83-7. doi: 10.1038/ng0994-83

5. Finnish-German APECED Consortium. An autoimmune disease, APECED, caused by mutations in a novel gene featuring two PHDtype zinc-finger domains. Nat Genet. (1997) 17:399-403. doi: 10.1038/ ng1297-399

6. Su MA, Anderson MS. Aire: an update. Curr Opin Immunol. (2004) 16:74652. doi: 10.1016/j.coi.2004.09.009

7. Ramsey C, Bukrinsky A, Peltonen L. Systematic mutagenesis of the functional domains of AIRE reveals their role in intracellular targeting. Hum Mol Genet. (2002) 11:3299-308. doi: $10.1093 / \mathrm{hmg} / 11.26 .3299$

8. Pereira LE, Bostik P, Ansari AA. The development of mouse APECED models provides new insight into the role of AIRE in immune regulation. Clin Dev Immunol. (2005) 12:211-6. doi: 10.1080/17402520500212589

9. Björses P, Aaltonen J, Vikman A, Perheentupa J, Ben-Zion G, Chiumello G, et al. Genetic homogeneity of autoimmune polyglandular disease type I. Am J Hum Genet. (1996) 59:879-86.

10. Björses P, Halonen M, Palvimo JJ, Kolmer M, Aaltonen J, Ellonen $\mathrm{P}$, et al. Mutations in the AIRE gene: effects on subcellular location and transactivation function of the autoimmune promotes epithelial barrier integrity, especially in synergy with TNF- $\alpha$ co-secreted by Th22 cells (92). Moreover, the production of IL-22 is severely impaired by skin-populating T-cells from APECED patients (93).

\section{CONCLUSION}

In APECED patients, CMC is associated with an impaired Th17 cell response. However, it remains unclear whether decreased serum IL-17 and IL-22 levels are related to a defect in cytokine production or to neutralizing autoantibodies resulting from mutations in the AIRE gene. Further investigations to develop new host- or pathogen-derived biomarkers are needed to improve the diagnosis of CMC and for a better understanding of human epithelial immunity against C. albicans infection.

\section{AUTHOR CONTRIBUTIONS}

BS and LH conceived the framework of the review. BS, LH, MC, $\mathrm{JB}$, and $\mathrm{M}-\mathrm{CV}$ wrote the manuscript. EP-L and J-LW revised the manuscript. LH and MC created the Figure. All authors have read and approved the final version of the manuscript.

\section{ACKNOWLEDGMENTS}

The authors would like to thank Gwendal Geoffroy who actively participated in the graphical improvement of Figure 1. polyendocrinopathy-candidiasis-ectodermal dystrophy protein. Am J Hum Genet. (2000) 66:378-92. doi: 10.1086/302765

11. Heino M, Peterson P, Kudoh J, Shimizu N, Antonarakis SE, Scott HS, et al. APECED mutations in the autoimmune regulator (AIRE) gene. Hum Mutat. (2001) 18:205-11. doi: 10.1002/humu.1176

12. Zhu W, Hu Z, Liao X, Chen X, Huang W, Zhong $\mathrm{Y}$, et al. A new mutation site in the AIRE gene causes autoimmune polyendocrine syndrome type 1. Immunogenetics (2017) 69:643-51 doi: 10.1007/s00251-017-0995-5

13. Ahonen P, Miettinen A, Perheentupa J. Adrenal and steroidal cell antibodies in patients with autoimmune polyglandular disease type I and risk of adrenocortical and ovarian failure. J Clin Endocrinol Metab. (1987) 64:494500. doi: 10.1210/jcem-64-3-494

14. Rosatelli MC, Meloni A, Meloni A, Devoto M, Cao A, Scott HS, et al. A common mutation in Sardinian autoimmune polyendocrinopathycandidiasis-ectodermal dystrophy patients. Hum Genet. (1998) 103:428-34.

15. Zlotogora J, Shapiro MS. Polyglandular autoimmune syndrome type I among Iranian Jews. J Med Genet. (1992) 29:824-6.

16. Pearce SH, Cheetham T, Imrie H, Vaidya B, Barnes ND, Bilous RW, et al. A common and recurrent 13-bp deletion in the autoimmune regulator gene in British kindreds with autoimmune polyendocrinopathy type 1. Am J Hum Genet. (1998) 63:1675-84. doi: 10.1086/302145

17. Myhre AG, Halonen M, Eskelin P, Ekwall O, Hedstrand H, Rorsman F, et al. Autoimmune polyendocrine syndrome type 1 (APS I) in Norway. Clin Endocrinol. (2001) 54:211-7. doi: 10.1046/j.1365-2265.2001.01201.x

18. Dominguez M, Crushell E, Ilmarinen T, McGovern E, Collins S, Chang $\mathrm{B}$, et al. Autoimmune polyendocrinopathy-candidiasis-ectodermal dystrophy (APECED) in the Irish population. J Pediatr Endocrinol Metab. (2006) 19:1343-52. doi: 10.1515/JPEM,2006.19.11.1343

19. Wolff ASB, Erichsen MM, Meager A, Magitta NF, Myhre AG, Bollerslev J, et al. Autoimmune polyendocrine syndrome type 1 in Norway: phenotypic variation, autoantibodies, and novel mutations in the autoimmune regulator 
gene. J Clin Endocrinol Metab. (2007) 92:595-603. doi: 10.1210/jc.20 06-1873

20. Valenzise M, Fierabracci A, Cappa M, Porcelli P, Barcellona R, De Luca F, et al. Autoimmune polyendocrinopathy-candidiasis-ectodermal dystrophy: report of seven additional sicilian patients and overview of the overall series from sicily. Horm Res Paediatr. (2014) 82:127-32. doi: 10.1159/0003 63537

21. Ferre EM, Rose SR, Rosenzweig SD, Burbelo PD, Romito KR, Niemela JE, et al. Redefined clinical features and diagnostic criteria in autoimmune polyendocrinopathy-candidiasis-ectodermal dystrophy. JCI Insight (2016) 1:13. doi: $10.1172 /$ jci.insight. 88782

22. Halonen M, Eskelin P, Myhre A-G, Perheentupa J, Husebye ES, Kämpe $\mathrm{O}$, et al. AIRE mutations and human leukocyte antigen genotypes as determinants of the autoimmune polyendocrinopathy-candidiasisectodermal dystrophy phenotype. J Clin Endocrinol Metab. (2002) 87:2568-74. doi: $10.1210 /$ jcem.87.6.8564

23. Perheentupa J. Autoimmune polyendocrinopathy-candidiasisectodermal dystrophy. J Clin Endocrinol Metab. (2006) 91:2843-50. doi: 10.1210/jc.2005-2611

24. Zhu F, Willette-Brown J, Song N-Y, Lomada D, Song Y, Xue $\mathrm{L}$, et al. Autoreactive $\mathrm{T}$ cells and chronic fungal infection drive esophageal carcinogenesis. Cell Host Microbe (2017) 21:478-93.e7. doi: 10.1016/j.chom.2017.03.006

25. Eyerich K, Eyerich S, Hiller J, Behrendt H, Traidl-Hoffmann C. Chronic mucocutaneous candidiasis, from bench to bedside. Eur J Dermatol. (2010) 20:260-5. doi: 10.1684/ejd.2010.0910

26. Thorpe ES, Handley HE. Chronic tetany and chronic mycelial stomatitis in a child aged four and one-half years. Am J Dis Child (1929) 38:328-38. doi:10.1001/archpedi.1929.01930080104011

27. de Repentigny L, Lewandowski D, Jolicoeur P. Immunopathogenesis of oropharyngeal candidiasis in human immunodeficiency virus infection. Clin Microbiol Rev. (2004) 17:729-59. doi: 10.1128/CMR.17.4.729759.2004 .

28. Cornely OA, Bassetti M, Calandra T, Garbino J, Kullberg BJ, Lortholary O, et al. ESCMID* guideline for the diagnosis and management of Candida diseases 2012: non-neutropenic adult patients. Clin Microbiol Infect. (2012) 18:19-37. doi: 10.1111/1469-0691.12039

29. Rosa DD, Pasqualotto AC, Denning DW. Chronic mucocutaneous candidiasis and oesophageal cancer. Med Mycol. (2008) 46:85-91. doi: 10.1080/13693780701616023.

30. Mohammadi F, Javaheri MR, Nekoeian S, Dehghan P. Identification of Candida species in the oral cavity of diabetic patients. Curr Med Mycol. (2016) 2:1-7.doi: 10.18869/acadpub.cmm.2.2.4

31. Puel A, Cypowyj S, Maródi L, Abel L, Picard C, Casanova J-L. Inborn errors of human IL-17 immunity underlie chronic mucocutaneous candidiasis. Curr Opin Allergy Clin Immunol. (2012) 12:616. doi: 10.1097/ACI.0b013e328358cc0b.

32. Picard C, Al-Herz W, Bousfiha A, Casanova J-L, Chatila T, Conley ME, et al. Primary immunodeficiency diseases: an update on the classification from the International Union of Immunological Societies Expert Committee for Primary Immunodeficiency 2015. J Clin Immunol. (2015) 35:696-726. doi: 10.1007/s10875-015-0201-1.

33. Okada S, Puel A, Casanova J-L, Kobayashi M. Chronic mucocutaneous candidiasis disease associated with inborn errors of IL-17 immunity. Clin Transl Immunol. (2016) 5:e114. doi: 10.1038/cti.2016.71

34. Sowerwine KJ, Holland SM, Freeman AF. Hyper-IgE syndrome update. Ann N Y Acad Sci. (2012) 1250:25-32. doi: 10.1111/j.1749-6632.2011.06387.x

35. Zhang Q, Davis JC, Lamborn IT, Freeman AF, Jing H, Favreau $\mathrm{AJ}$, et al. Combined Immunodeficiency Associated with DOCK8 Mutations. N Engl J Med. (2009) 361:2046-55. doi: 10.1056/NEJMoa 0905506

36. Su HC. DOCK8 (Dedicator of cytokinesis 8) deficiency. Curr Opin Allergy Clin Immunol. (2010) 10:515-20. doi: 10.1097/ACI.0b013e32833fd718

37. Su HC, Jing H, Zhang Q. DOCK8 deficiency. Ann N Y Acad Sci. (2011) 1246:26-33. doi: 10.1111/j.1749-6632.2011.06295.x

38. Biggs CM, Keles S, Chatila TA. DOCK8 deficiency: insights into pathophysiology, clinical features and management. Clin Immunol. (2017) 181:75-82. doi: 10.1016/j.clim.2017.06.003
39. Grumach AS, de Queiroz-Telles F, Migaud M, Lanternier F, Filho NR, Palma SMU, et al. A homozygous CARD9 mutation in a Brazilian patient with deep dermatophytosis. J Clin Immunol. (2015) 35:486-90. doi: 10.1007/s10875-015-0170-4

40. Celmeli F, Oztoprak N, Turkkahraman D, Seyman D, Mutlu E, Frede N, et al. Successful granulocyte colony-stimulating factor treatment of relapsing Candida albicans meningoencephalitis caused by CARD9 deficiency. Pediatr Infect Dis J. (2016) 35:428-31. doi: 10.1097/INF.00000000000 01028

41. Jones N, Garcez T, Newman W, Denning D. Endogenous Candida endophthalmitis and osteomyelitis associated with CARD9 deficiency. BMJ Case Rep (2016) 2016:bcr2015214117. doi: 10.1136/bcr-2015-214117. [Epub ahead of print].

42. Glocker E-O, Hennigs A, Nabavi M, Schäffer AA, Woellner C, Salzer U, et al. A homozygous CARD9 mutation in a family with susceptibility to fungal infections. N Engl J Med. (2009) 361:1727-35. doi: 10.1056/NEJMoa08 10719

43. Drewniak A, Gazendam RP, Tool ATJ, van Houdt M, Jansen MH, van Hamme JL, et al. Invasive fungal infection and impaired neutrophil killing in human CARD9 deficiency. Blood (2013) 121:2385-92. doi: 10.1182/blood-2012-08-450551

44. Ferwerda B, Ferwerda G, Plantinga TS, Willment JA, van Spriel AB, Venselaar H, et al. Human Dectin-1 deficiency and mucocutaneous fungal infections. N Engl J Med. (2009) 361:1760-7. doi: 10.1056/NEJMoa09 01053.

45. Rosentul DC, Plantinga TS, Oosting M, Scott WK, Velez Edwards DR, Smith PB, et al. Genetic variation in the Dectin-1/CARD9 recognition pathway and susceptibility to candidemia. J Infect Dis. (2011) 204:1138-45. doi: 10.1093/infdis/jir458.

46. Plantinga TS, van der Velden WJFM, Ferwerda B, van Spriel AB, Adema G, Feuth T, et al. Early stop polymorphism in human DECTIN-1 is associated with increased Candida colonization in hematopoietic stem cell transplant recipients. Clin Infect Dis. (2009) 49:724-32. doi: 10.1086/ 604714

47. Saijo S, Ikeda S, Yamabe K, Kakuta S, Ishigame H, Akitsu A, et al. Dectin2 recognition of alpha-mannans and induction of Th17 cell differentiation is essential for host defense against Candida albicans. Immunity (2010) 32:68191. doi: 10.1016/j.immuni.2010.05.001

48. Vinh DC. Insights into human antifungal immunity from primary immunodeficiencies. Lancet Infect Dis. (2011) 11:780-92. doi: 10.1016/S1473-3099(11)70217-1

49. Hueber W, Sands BE, Lewitzky S, Vandemeulebroecke M, Reinisch W, Higgins PDR, et al. Secukinumab, a human anti-IL-17A monoclonal antibody, for moderate to severe Crohn's disease: unexpected results of a randomised, double-blind placebo-controlled trial. Gut (2012) 61:1693-700. doi: 10.1136/gutjnl-2011-301668

50. Colombel JF, Sendid B, Jouault T, Poulain D. Secukinumab failure in Crohn's disease: the yeast connection? Gut (2013) 62:800-1. doi: 10.1136/gutjnl-2012-304154

51. Ahonen P, Myllärniemi S, Sipilä I, Perheentupa J. Clinical variation of Autoimmune Polyendocrinopathy-Candidiasis-Ectodermal Dystrophy (APECED) in a series of 68 patients. N Engl J Med. (1990) 322:1829-36. doi: 10.1056/NEJM19900628322260

52. Collins SM, Dominguez $\mathrm{M}$, Ilmarinen $\mathrm{T}$, Costigan $\mathrm{C}$, Irvine $\mathrm{AD}$. Dermatological manifestations of autoimmune polyendocrinopathycandidiasis-ectodermal dystrophy syndrome. Br J Dermatol. (2006) 154:1088-93. doi: 10.1111/j.1365-2133.2006.07166.x

53. Bruserud $\varnothing$, Oftedal BE, Landegren N, Erichsen MM, Bratland E, Lima $\mathrm{K}$, et al. A longitudinal follow-up of autoimmune polyendocrine syndrome type 1. J Clin Endocrinol Metab. (2016) 101:2975-83. doi: 10.1210/jc.201 6-1821

54. Betterle C, Greggio NA, Volpato M. Autoimmune polyglandular syndrome type 1. J Clin Endocrinol Metab. (1998) 83:1049-55. doi: $10.1210 /$ jcem.83.4.4682

55. Meloni A, Willcox N, Meager A, Atzeni M, Wolff ASB, Husebye ES, et al. Autoimmune polyendocrine syndrome type 1: an extensive longitudinal study in sardinian patients. J Clin Endocrinol Metab. (2012) 97:1114-24. doi: 10.1210/jc.2011-2461 
56. Husebye ES, Perheentupa J, Rautemaa R, Kämpe O. Clinical manifestations and management of patients with autoimmune polyendocrine syndrome type I. J Intern Med. (2009) 265:514-29. doi: 10.1111/j.1365-2796.2009. 02090.x

57. Proust-Lemoine E, Saugier-Véber P, Lefranc D, Dubucquoi S, Ryndak A, Buob D, et al. Autoimmune polyendocrine syndrome type 1 in North-Western France: AIRE gene mutation specificities and severe forms needing immunosuppressive therapies. Horm Res Paediatr. (2010) 74:275-84. doi: $10.1159 / 000297714$

58. Puel A, Döffinger R, Natividad A, Chrabieh M, Barcenas-Morales G, Picard C, et al. Autoantibodies against IL-17A, IL-17F, and IL-22 in patients with chronic mucocutaneous candidiasis and autoimmune polyendocrine syndrome type I. J Exp Med. (2010) 207:291-7. doi: 10.1084/jem.20091983.

59. Kisand K, Wolff ASB, Podkrajšek KT, Tserel L, Link M, Kisand KV, et al. Chronic mucocutaneous candidiasis in APECED or thymoma patients correlates with autoimmunity to Th17-associated cytokines. J Exp Med. (2010) 207:299-308. doi: 10.1084/jem.20091669

60. Rautemaa R, Hietanen J, Niissalo S, Pirinen S, Perheentupa J. Oral and oesophageal squamous cell carcinoma - A complication or component of autoimmune polyendocrinopathy-candidiasisectodermal dystrophy (APECED, APS-I). Oral Oncol. (2007) 43:607-13. doi: 10.1016/j.oraloncology.2006.07.005

61. Böckle BC, Wilhelm M, Müller H, Götsch C, Sepp NT. Oral mucous squamous cell carcinoma-an anticipated consequence of autoimmune polyendocrinopathy-candidiasis-ectodermal dystrophy (APECED). J Am Acad Dermatol. (2010) 62:864-8. doi: 10.1016/j.jaad.2009. 06.061

62. Koo S, Kejariwal D, Al-Shehri T, Dhar A, Lilic D. Oesophageal candidiasis and squamous cell cancer in patients with gain-of-function STAT1 gene mutation. United Eur Gastroenterol J. (2017) 5:625-31. doi: 10.1177/20506406166 84404

63. Richman RA, Rosenthal IM, Solomon LM, Karachorlu KV. Candidiasis and multiple endocrinopathy: with oral squamous cell carcinoma complications. Arch Dermatol. (1975) 111:625-7.

64. Chi AC, Day TA, Neville BW. Oral cavity and oropharyngeal squamous cell carcinoma-an update. CA Cancer J Clin. (2015) 65:401-21. doi: $10.3322 /$ caac. 21293

65. Shephard MK, Schifter M, Palme CE. Multiple oral squamous cell carcinomas associated with autoimmune polyendocrinopathy-candidiasis-ectodermal dystrophy. Oral Surg Oral Med Oral Pathol Oral Radiol. (2012) 114:e36-42. doi: 10.1016/j.0ooo.2012.04.013

66. Binnie WH, Rankin KV, Mackenzie IC. Etiology of oral squamous cell carcinoma. J Oral Pathol. (1983) 12:11-29.

67. Mehrotra R, Yadav S. Oral squamous cell carcinoma: etiology, pathogenesis and prognostic value of genomic alterations. Indian J Cancer (2006) 43:60-6. doi: 10.4103/0019-509X.25886

68. Swidergall M, Solis NV, Lionakis MS, Filler SG. EphA2 is an epithelial cell pattern recognition receptor for fungal $\beta$-glucans. Nat Microbiol. (2018) 3:53-61. doi: 10.1038/s41564-017-0059-5

69. Zhu W, Phan QT, Boontheung P, Solis NV, Loo JA, Filler SG. EGFR and HER2 receptor kinase signaling mediate epithelial cell invasion by Candida albicans during oropharyngeal infection. Proc Natl Acad Sci USA. (2012) 109:14194-9. doi: 10.1073/pnas.1117676109

70. Rex JH, Rinaldi MG, Pfaller MA. Resistance of Candida species to fluconazole. Antimicrob Agents Chemother. (1995) 39:1-8.

71. Heald AE, Cox GM, Schell WA, Bartlett JA, Perfect JR. Oropharyngeal yeast flora and fluconazole resistance in HIV-infected patients receiving long-term continuous versus intermittent fluconazole therapy. AIDS (1996) 10:263-8.

72. Rautemaa R, Richardson M, Pfaller M, Perheentupa J, Saxén H. Reduction of fluconazole susceptibility of Candida albicans in APECED patients due to long-term use of ketoconazole and miconazole. Scand J Infect Dis. (2008) 40:904-7. doi: 10.1080/00365540802275853

73. Rautemaa R, Richardson M, Pfaller MA, Perheentupa J, Saxén H. Activity of amphotericin B, anidulafungin, caspofungin, micafungin, posaconazole, and voriconazole against Candida albicans with decreased susceptibility to fluconazole from APECED patients on long-term azole treatment of chronic mucocutaneous candidiasis. Diagn Microbiol Infect Dis. (2008) 62:182-5. doi: 10.1016/j.diagmicrobio.2008.05.007
74. McManus BA, McGovern E, Moran GP, Healy CM, Nunn J, Fleming $\mathrm{P}$, et al. Microbiological screening of irish patients with autoimmune polyendocrinopathy-candidiasis-ectodermal dystrophy reveals persistence of Candida albicans Strains, gradual reduction in susceptibility to azoles, and incidences of clinical signs of oral candidiasis without culture evidence. J Clin Microbiol. (2011) 49:1879-89. doi: 10.1128/JCM.00026-11

75. Proust-Lemoine E, Guyot S. Polyendocrinopathies auto-immunes de type 1 et pathologies buccales. Presse Méd. (2017) 46:853-63. doi: 10.1016/j.lpm.2017.05.029

76. Rautemaa R, Richardson M, Pfaller M, Koukila-Kähkölä P, Perheentupa J, Saxén H. Decreased susceptibility of Candida albicans to azole antifungals: a complication of long-term treatment in autoimmune polyendocrinopathy-candidiasis-ectodermal dystrophy (APECED) patients. J Antimicrob Chemother. (2007) 60:889-92. doi: 10.1093/jac/ dkm299

77. Siikala E, Rautemaa R, Richardson M, Saxen H, Bowyer P, Sanglard D. Persistent Candida albicans colonization and molecular mechanisms of azole resistance in autoimmune polyendocrinopathy-candidiasis-ectodermal dystrophy (APECED) patients. J Antimicrob Chemother. (2010) 65:2505-13. doi: $10.1093 / \mathrm{jac} / \mathrm{dkq} 354$

78. Galeotti C, Kaveri SV, Bayry J. IVIG-mediated effector functions in autoimmune and inflammatory diseases. Int Immunol. (2017) 29:491-8. doi: $10.1093 /$ intimm/dxx039

79. Bayry J, Lacroix-Desmazes S, Kazatchkine MD, Kaveri SV. Intravenous immunoglobulin for infectious diseases: back to the pre-antibiotic and passive prophylaxis era? Trends Pharmacol Sci. (2004) 25:306-10. doi: 10.1016/j.tips.2004.04.002

80. Ferrara G, Zumla A, Maeurer M. Intravenous immunoglobulin (IVIg) for refractory and difficult-to-treat infections. Am J Med. (2012) 125:1036.e1-8. doi: 10.1016/j.amjmed.2012.01.023

81. Maddur MS, Vani J, Hegde P, Lacroix-Desmazes S, Kaveri SV, Bayry J. Inhibition of differentiation, amplification, and function of human TH17 cells by intravenous immunoglobulin. J Allergy Clin Immunol. (2011) 127:823-30. doi: 10.1016/j.jaci.2010.12.1102

82. Saha C, Das M, Patil V, Stephen-Victor E, Sharma M, Wymann S, et al. Monomeric immunoglobulin A from plasma inhibits human Th17 responses in vitro independent of Fc $\alpha$ RI and DC-SIGN. Front Immunol. (2017) 8:275. doi: 10.3389/fimmu.2017.00275

83. Bozza S, Käsermann F, Kaveri SV, Romani L, Bayry J. Intravenous immunoglobulin protects from experimental allergic bronchopulmonary aspergillosis via a sialylation-dependent mechanism. Eur J Immunol. (2018). doi: 10.1002/eji.201847774. [Epub ahead of print].

84. Meager A, Visvalingam K, Peterson $\mathrm{P}$, Möll K, Murumägi A, Krohn K, et al. Anti-interferon autoantibodies in autoimmune polyendocrinopathy syndrome type 1. PLoS Med. (2006) 3:e289. doi: 10.1371/journal.pmed.0030289

85. Meloni A, Furcas M, Cetani F, Marcocci C, Falorni A, Perniola R, et al. Autoantibodies against type I interferons as an additional diagnostic criterion for autoimmune polyendocrine syndrome type I. J Clin Endocrinol Metab. (2008) 93:4389-97. doi: 10.1210/jc.2008-0935

86. Kisand K, Lilic D, Casanova J-L, Peterson P, Meager A, Willcox N. Mucocutaneous candidiasis and autoimmunity against cytokines in APECED and thymoma patients: clinical and pathogenetic implications. Eur J Immunol. (2011) 41:1517-27. doi: 10.1002/eji.201041253

87. Sarkadi AK, Taskó S, Csorba G, Tóth B, Erdos M, Maródi L. Autoantibodies to IL-17A may be Correlated with the Severity of Mucocutaneous Candidiasis in APECED Patients. J Clin Immunol. (2014) 34:181-93. doi: 10.1007/s10875-014-9987-5

88. Bichele R, Kärner J, Truusalu K, Smidt I, Mändar R, Conti HR, et al. IL-22 neutralizing autoantibodies impair fungal clearance in murine oropharyngeal candidiasis model. Eur J Immunol. (2018) 48:464-70. doi: 10.1002/eji.201747209

89. Kisand K, Link M, Wolff ASB, Meager A, Tserel L, Org T, et al Interferon autoantibodies associated with AIRE deficiency decrease the expression of IFN-stimulated genes. Blood (2008) 112:2657-66. doi: 10.1182/blood-2008-03-144634

90. Ng WF, von Delwig A, Carmichael AJ, Arkwright PD, Abinun $\mathrm{M}$, Cant AJ, et al. Impaired TH17 responses in patients with 
chronic mucocutaneous candidiasis with and without autoimmune polyendocrinopathy-candidiasis-ectodermal dystrophy. J Allergy Clin Immunol. (2010) 126:1006-15. doi: 10.1016/j.jaci.2010. 08.027

91. Ahlgren KM, Moretti S, Lundgren BA, Karlsson I, Åhlin E, Norling A, et al. Increased IL-17A secretion in response to Candida albicans in autoimmune polyendocrine syndrome type 1 and its animal model. Eur J Immunol. (2011) 41:235-45. doi: 10.1002/eji.200939883

92. Kisand K, Peterson P. Autoimmune polyendocrinopathy candidiasis ectodermal dystrophy. J Clin Immunol. (2015) 35:463-78. doi: 10.1007/s10875-015-0176-y

93. Laakso SM, Kekäläinen E, Heikkilä N, Mannerström H, Kisand K, Peterson $\mathrm{P}$, et al. In vivo analysis of helper $\mathrm{T}$ cell responses in patients with autoimmune polyendocrinopathy - candidiasis - ectodermal dystrophy provides evidence in support of an IL-22 defect. Autoimmunity (2014) 47:55662. doi: 10.3109/08916934.2014.929666.

Conflict of Interest Statement: The authors declare that the research was conducted in the absence of any commercial or financial relationships that could be construed as a potential conflict of interest.

Copyright (c) 2018 Humbert, Cornu, Proust-Lemoine, Bayry, Wemeau, Vantyghem and Sendid. This is an open-access article distributed under the terms of the Creative Commons Attribution License (CC BY). The use, distribution or reproduction in other forums is permitted, provided the original author(s) and the copyright owner(s) are credited and that the original publication in this journal is cited, in accordance with accepted academic practice. No use, distribution or reproduction is permitted which does not comply with these terms. 